\title{
Deformation mechanism under essential work of fracture process in polycyclo-olefin materials
}

\author{
T. Kuno, Y. Yamagishi, T. Kawamura, K. Nitta* \\ Division of Material Sciences, Graduate School of Natural Sciences and Technology, Kanazawa University, Kanazawa, \\ 920-1192 Japan
}

Received 8 February 2008; accepted in revised form 11 April 2008

\begin{abstract}
The fracture toughness of a glassy polycyclo-olefin (PCO) was investigated by the essential work of fracture (EWF) method using a double-edge notched specimens. It was shown that the PCO follows the EWF concept in the temperature range between room temperature and glass transition temperature $T_{g}$ where the ligament yielding appear at a maximum point on the stress-displacement curves and subsequently the necking and tearing processes take place in the post yielding region. The essential work of fracture required for the ligament yielding drops as the temperature approaches $T_{g}$. The non-essential work of fracture attributed to tearing process after yielding is consumed to expand the plastic region and causes molecular chains to orient to the stretching direction.
\end{abstract}

Keywords: fracture and fatigue, essential work of fracture (EWF), polycyclo-olefin, crack opening displacement, rheooptics

\section{Introduction}

Polycyclo-olefin (PCO) is a novel type of glassy polymers and received special attention in optical applications because of its environmental friendly and excellent heat- and chemical-resistances in addition to considerably low bifringence and high transparency. According to our previous studies on its tensile properties [1], PCO materials exhibited a high ductility near their glass transition temperatures $T_{g}$ at which the PCOs are carefully compression molded to produce transparent sheets and films. The mechanical data in the vicinity of $T_{g}$ are very useful to optimize the molding conditions.

The essential work of fracture (EWF) method developed by Broberg [2] has been conveniently used for the examination of fracture properties of ductile thin films. When a double edged notch specimen is loaded under uniaxial tension, the fracture is dissipated in two distinct zones such as frac- ture process zone (or essential zone) and plastic zone (or non-essential zone). The total work of fracture $W_{f}$ is expressed as the sum between the two parameters of the essential work $W_{e}$ and the nonessential work $W_{p}$, as shown by Equation (1) [2-6]:

$$
W_{f}=W_{e}+W_{p}
$$

The first term is related to the instability of the crack tip and represents the energy required to fracture the polymer in its process zone which is surface-related whereas the second term is associated with the plastic work, which is proportional to the plastic zone volume, and represents the energy consumed by various deformation mechanisms in the surrounding outer plastic zone [7-9]. When the typical load-displacement curve of double-notched specimen shows a maximum point at which the ligament region is fully yielded and the yielding region is necked after maximum, then may be parti-

*Corresponding author, e-mail: nitta@t.kanazawa-u.ac.jp

(c) BME-PT and GTE 


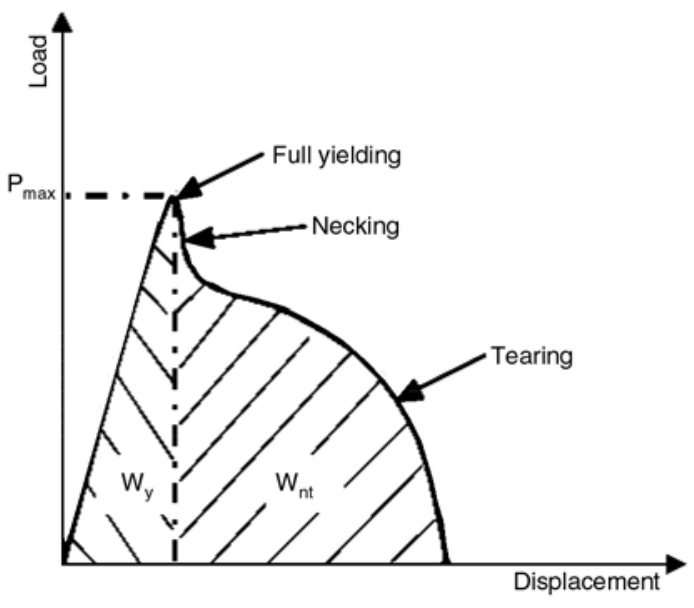

Figure 1. Schematic explanation of the definition of $W_{y}$ and $W_{n t}$

tioned into two components as illustrated in Figure 1. Consequently, we have Equation (2):

$W_{f}=W_{y}+W_{n t}$

where $W_{y}$ is the mechanical energy required for yielding of the ligament region and $W_{n t}$ is the energy required for necking and subsequent tearing.

The EWF method has been widely applied to various commodity plastics such as poly(ethylene terephthalate), polyethylene, polypropylene, and their blends. It has been proven theoretically and confirmed experimentally that the essential work of fracture corresponds to the critical J-integral value which is the reliable method for characterizing the fracture toughness of ductile polymeric materials $[10,11]$. The present work applies the concept of EWF for characterizing ductile fracture of PCO films as a function of temperature.

\section{Analytical methods for essential work of fracture}

The energy required for fracture $W_{e}$ can be considered to be a pure crack resistance parameter. As a result, the value of $W_{e}$ is essentially a surface energy and is proportional to the ligament length $L$ for a given specimen thickness $t$. The plastic work $W_{p}$ is a volume energy, which involves microvoiding and shear yielding and it is proportional to the volume $L^{2} t$. Dividing Equation (1) by the ligament section $L t$, we have Equation (3):

$$
w_{f}=\frac{W_{f}}{L t}=w_{e}+\beta w_{p} L
$$

where $\beta$ is a shape factor associated with the volume of the plastic deformation zone, and $w_{p}$ is the specific non-essential work of fracture, which is the plastic work per unit volume of the plastic deformation zone, and the specific essential work $w_{e}$ is the failure work per unit surface area.

Equation (3) indicates that $w_{f}$ is a linear function of ligament length $L$ where the specific essential work of fracture $w_{e}$ is given by the positive intercept at $L=0$ of the linear regression interpolating the data and the specific non-essential work of fracture $\beta w_{p}$ is given by the slope of the regression line. The main objective of the EWF method is to determine $w_{e}$ as a toughness parameter.

The specific essential work of fracture $w_{e}$ can be considered as a material constant under plane stress conditions for a given thickness $t$, as suggested from Equation (3). Furthermore, it is significant to consider partitioning of $w_{e}$ since the total fracture work can be partitioned into two components of the ligament yielding and tearing work according to Equation (2) [12-14]. Consequently, the following equations have the form (see Equations (4) and (5)):

$w_{e}=w_{e y}+w_{e n t}$

$\beta w_{p}=\beta_{y} w_{p y}+\beta_{n t} w_{p n t}$

where $w_{e y}$ is the specific essential yielding-related work of fracture, which is considered to be a more reliable parameter as compared to $w_{e}$ to follow the extrinsic effects of the physical aging, the loading of plasticizer, and the water content [15-18], $w_{\text {ent }}$ is the specific essential tearing work, $w_{p y}$ is the volumetric energy dissipated during yielding, and $w_{p n t}$ is the dissipated work during tearing, and $\beta_{y}$ and $\beta_{n t}$ are geometric factors related to the shape of the plastic zone during the yielding and tearing stages, respectively.

\section{Experimental}

\subsection{Materials}

Polycyclo-olefin (Commercial grade: ZEONOR 1600) was used in this study. The PCO samples 
were compression molded to sheets of about $100 \mu \mathrm{m}$ thickness for 10 minutes under $270^{\circ} \mathrm{C}$ and $20 \mathrm{MPa}$. The rectangular sheets were notched to produce double-edge-notched tension (DENT) specimens with various ligament lengths by way of forming double edge notch transverse to the elongation direction by a sharp razor.

\subsection{Standard EWF fracture tests}

The DENT specimens with $20 \mathrm{~mm}$ width were tested to complete failure in an Instron 4466 tensile machine equipped maximum $100 \mathrm{~N}$ load cell and an isothermal chamber. The tests were performed at a fixed elongation rate of $1 \mathrm{~mm} / \mathrm{min}$ between 15 and $163^{\circ} \mathrm{C}$. The initial distance between cramps was $10 \mathrm{~mm}$. We performed the EWF fracture measurements using more than 6 pieces with different ligament lengths ranging from 2.5 to $15 \mathrm{~mm}$ for each temperature. The mechanical energies $W_{y}$ and $W_{n t}$ were evaluated from the integration of the resulting load-displacement curves (see Figure 1).

\subsection{Rheo-optical EWF fracture tests}

Rheo-optical techniques [19] afford information on the elongation time dependence not only of the stress but also of optical quantities associated directly with the structure. In this study, infrared dichroism was measured simultaneously with stress during EWF tests at a constant rate of elongation.

For the purpose, a hand-made tensile tester was set in a Fourier-transformation infrared spectroscopic analyzer (FT-IR: MIR-8000 of ORIEL Co.) in such a way as to allow infrared polarized beam go through a DENT specimen mounted on the tensile tester. The tensile tester was specially designed for upper and lower clamps to symmetrically move from the central point in the ligament zone of the film so that the IR beam spot ( $3 \mathrm{~mm}$ diameter) remains at the initial position during whole stretching [20]. The rheo-optical EWF tests were performed under a constant elongation rate of $1 \mathrm{~mm} / \mathrm{min}$. In addition, the sample chamber of the stretching machine was equipped with an adiabatic temperature cell to maintain the temperature control at $\pm 0.5^{\circ} \mathrm{C}$. The smaller DENT specimens with a width of $15 \mathrm{~mm}$ were used for the rheo-optical measurements because of the space limitation of the sample chamber.
To determine the orientation function of molecular chain axes, we used the dichroic ratio $D$ which can be determined by $A_{\|} / A_{\perp}$ where $A_{\|}$and $A_{\perp}$ denote the absorbances measured for the radiation whose electric vectors are parallel and perpendicular to the stretching direction, respectively. The orientation function of molecular chain $f$ is related to the dichroic ratio by Equation (6) [21, 22]:

$$
f=\frac{2}{3 \cos ^{2} \phi-1} \frac{D-1}{D+2}
$$

where the $\phi$ is the angle between the direction of the transition moment of the absorption band and the main chain axis.

The intensities of $1480 \mathrm{~cm}^{-1}$ absorption bands were measured as a function of elongation time or displacement every 1-2 s. The band is associated with $\mathrm{CH}_{2}$ scissoring vibration. The transition moment is considered to be 90 degrees direction against the main chain, $\phi=\pi / 2$. The $\mathrm{CH}_{2}$ scissoring of $\mathrm{PCO}$ was confirmed with the functional method of density using the calculation software of Gaussian Co. on the basis of a norbornene molecular model.

\subsection{Sample characterization}

The dynamic mechanical properties, the storage modulus $E^{\prime}$ and the loss modulus $E^{\prime \prime}$, were investigated in the tensile mode using the dynamic mechanical analyzer (DVE-V4 of Rheology Co.). The rectangular specimens with $7 \mathrm{~mm}$ width, $30 \mathrm{~mm}$ length, and $100 \mu \mathrm{m}$ thickness were used for the measurements. The testing condition is the strain amplitude of $0.1 \%$, the frequency $10 \mathrm{~Hz}$,

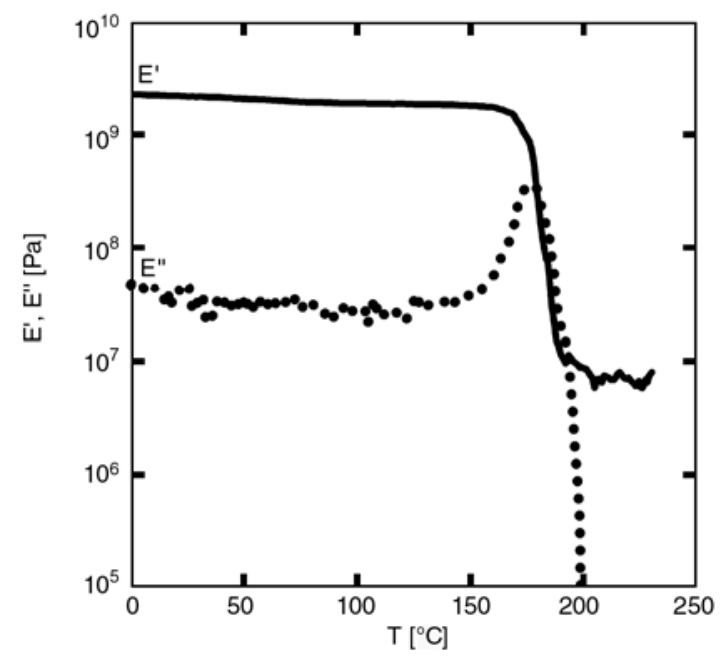

Figure 2. Dynamic mechanical spectra of PCO measured at $10 \mathrm{~Hz}$ 
heating rate $2^{\circ} \mathrm{C} / \mathrm{min}$ and the temperature range of $0-230^{\circ} \mathrm{C}$. The dynamic mechanical spectrum was exemplified in Figure 2. A sharp relaxation peak in $E^{\prime \prime}$ curve, ascribed to the glass transition $T_{g}$, appears at $175^{\circ} \mathrm{C}$ and the storage modulus $E^{\prime}$ sharply reduces around the temperature.

The uniaxial tensile tests of the PCO sample were carried out using the notched-type specimens at the same temperature as the standard EWF tests. Details of the tensile measurements are referred in our previous paper [1].

\section{Results and discussion}

Figure 3 exemplifies the load-displacement curves from the EWF measurements at $102^{\circ} \mathrm{C}$ for the DENT samples having different ligament lengths. The curves have a clear maximum point and show a prominent load drop over the maximum point. The peak in the curve corresponds to the yielding around the ligament area. The sample specimen is subsequently necked and the failure is caused by ductile tearing of the ligament region after yielding. The rise in temperature reduces the overall maximum load and increases the elongation at break.

The important feature of the load-displacement curves for DENT specimens as a function of ligament length is their geometrical similarity. We directly confirmed that the width of the ductile deformation region in the samples after EWF tests was within the ligament length for the samples tested below $157^{\circ} \mathrm{C}$. In addition, the linearity of the $L$ dependence of $W_{f}$ is established except for the values at $157^{\circ} \mathrm{C}$ within the squared multiple corre-

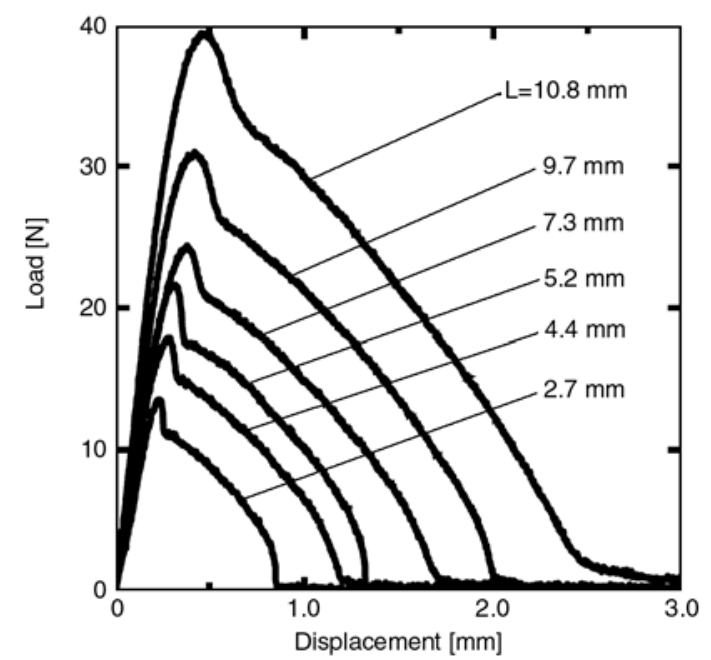

Figure 3. Force-displacement curves of the results of EWF test at $102^{\circ} \mathrm{C}$ lation coefficient $\left(R^{2}\right)$ more than 0.95 as shown in Figures 5. These results suggest that the experimental conditions in this study satisfy the basic requirement for the applicability of the EWF analysis $[6,14]$. Below room temperature, the failure of the PCO specimen occurs in a brittle manner in which the craze and/or cracks was formed at the root of the notch. Figure 4 shows the load-displacement curves measured at $15^{\circ} \mathrm{C}$, showing that the specimens were broken prior to necking being independent of the ligament length. In the temperature range from 50 to $157^{\circ} \mathrm{C}$, it was confirmed that the yielding of the ligament region occurs at maximum load at which two line plastic zones generated on the crack tips meet each other. The subsequent neck starts on the crack tips and rapidly reaches the whole ligament region, leading to a prominent load drop and then the crack starts to propagate across the necked zone until ultimate fracture occurs.

As shown in Figure 1, the specific work of fracture $w_{f}$, is the sum of their yielding component $w_{y}$ and the necking component $w_{n t}$. Figures $5 \mathrm{a}$ and $5 \mathrm{~b}$ show both components plotted against the ligament length $L$ where the values of $w_{y}$ and $w_{n t}$ were calculated by integration of the area under the forceelongation curves. We confirmed the linear relationship between the fracture works and the ligament length $L$.

The specific essential work of fracture parameters and the specific non-essential work can be determined from the linear interpolation of these plots. The intercept values at $L=0$ and slopes provide the essential work parameters and the non-essential work parameters, respectively, which were plotted

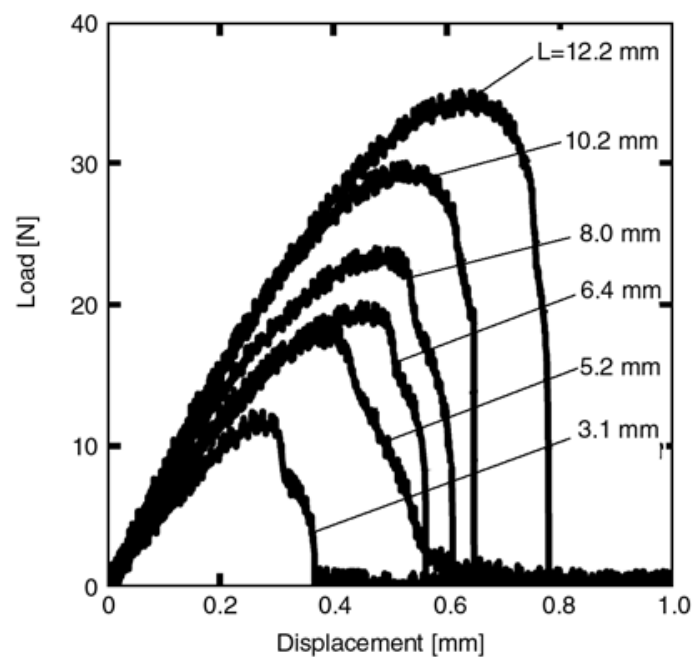

Figure 4. Force-displacement curves of the results of EWF test at $15^{\circ} \mathrm{C}$ 

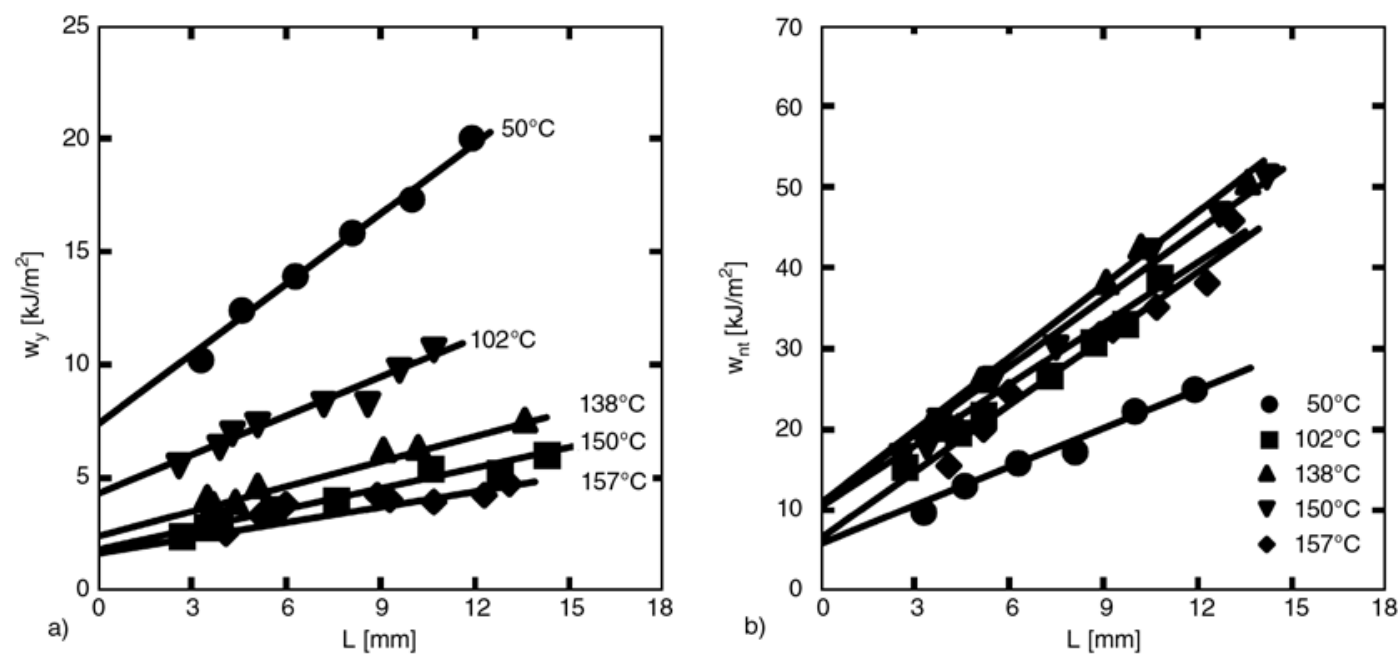

Figure 5. The dependence of $w_{y}$ (a) and $w_{n t}(\mathrm{~b})$ on the ligament length at various temperatures

as a function of temperature in Figures $6 a$ and $6 b$. The specific work of fracture $w_{e}$ is almost independent of temperature in the lower temperatures but decreases as the temperature approaches $T_{g}$. Its component $w_{e y}$ monotonically decreases with increasing temperature over the entire experimental temperature range whilst $w_{\text {ent }}$ shows a maximum and rapidly drops at near $T_{g}$. Similar results for PEEK samples were reported by Arkhireyeva et al. [14], demonstrating that the $w_{e y}$ values can be regarded as the specific essential work of fracture for crack initiation. Consequently, the variation of $w_{e}$ with temperature for PCO was found to be almost caused by the temperature dependence of crack initiation.

The values of specific non-essential work of fracture $\beta w_{p}, \beta_{y} w_{p y}$ and $\beta_{n t} w_{p n t}$ can be estimated from

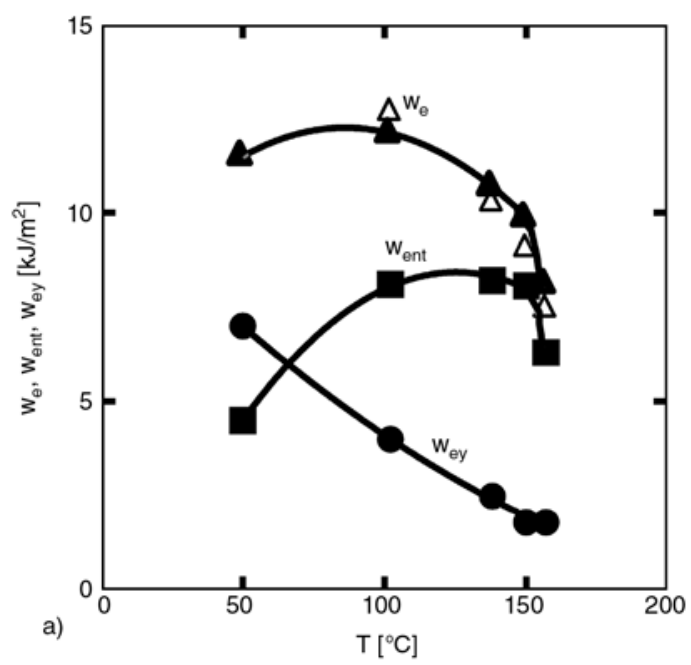

the slopes of the straight lines showed in Figures $5 \mathrm{a}$ and $5 \mathrm{~b}$. It was found that the values of $\beta w_{p}$ and $\beta_{n t} w_{p n t}$ increase with increasing temperature below $T_{g}$ and sharply drop at near $T_{g}$. On the other hand, the absolute values of the component $\beta_{y} w_{p y}$ were found to monotonously decrease with increasing the temperature. It should be noted here that the values of $\beta_{n t} w_{p n t}$ are much greater than those of $\beta_{y} w_{p y}$, indicating that almost non-essential work of fracture $\beta w_{p}$ is due to $\beta_{n t} w_{p n t}$ and the plastic zone development is mainly associated with the neckingtearing part of the fracture process.

The displacement at fracture $\delta_{b}$ can be divided into the yield displacement $\delta_{y}$ and tearing component $\delta_{n t}$ giving by $\delta_{b}=\delta_{y}+\delta_{n t}$. The values of $\delta_{b}, \delta_{y}$ and $\delta_{n t}$ are plotted against ligament length $L$ in Figures $7 \mathrm{a}-7 \mathrm{c}$. It was also found that the displace-

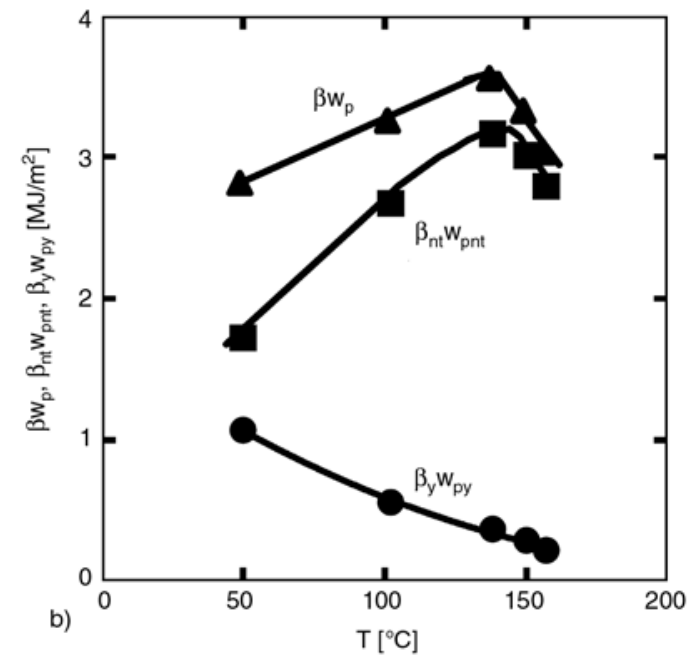

Figure 6. The temperature dependence of the essential work parameters (a) and the non-essential work parameters (b). The close symbols represent the parameters estimated directly from EWF tests and the open ones represent the parameters estimated from COD. 
ments $\delta_{b}, \delta_{y}$, and $\delta_{n t}$ represent the liner relationships with $L$ at any temperature. The displacement $\delta_{b}$ increases with increasing the temperature and
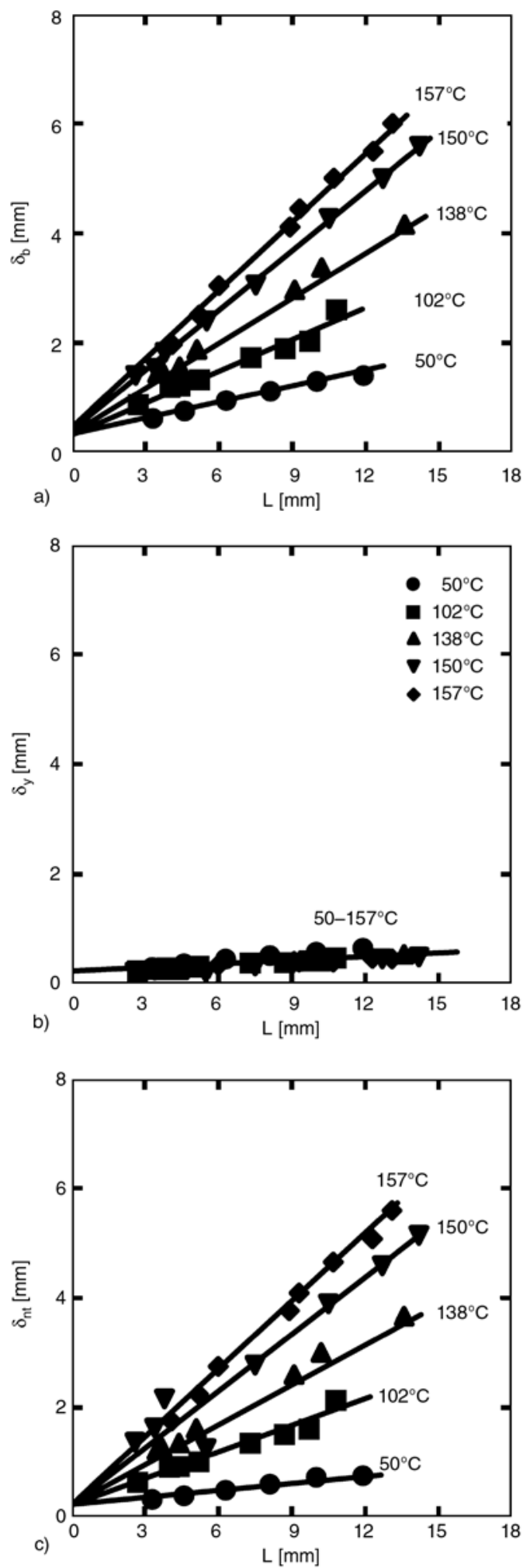

Figure 7. The dependence of displacement parameters on the ligament length at various temperatures. (a): total displacement $\delta_{b},(\mathrm{~b})$ : displacement of yielding region $\delta_{y}$ and (c): displacement of neckingtearing region $\delta_{n t}$ the yield displacement $\delta_{y}$ is slightly dependent on temperature as compared to the tearing displacement $\delta_{n t}$, indicating that the temperature sensitivity of the ultimate displacement $\delta_{b}$ is due to the temperature dependence of the tearing behavior. It is interesting to note that $\delta_{n t}$ and $\delta_{y}$ expolarate to a fixed value $(0.18 \mathrm{~mm})$ when $L \rightarrow 0$. Thus, we have the following empirical relations (see Equations (7) and (8)):

$\delta_{n t}=\delta_{e n t}+\alpha(T) L$

$\delta_{y}=\delta_{e y}+\alpha_{y} L$

The intercept value $\delta_{e}=\delta_{e n t}+\delta_{e y}=0.36 \mathrm{~mm}$ at $L=0$ has been identified as being equivalent to the critical crack opening displacement [23]. As seen in Figures 7, we have $\delta_{e n t}=\delta_{e y}=0.18 \mathrm{~mm}$ which were independent of temperature over the wide range from $50^{\circ} \mathrm{C}$ to near $T_{g}$, suggesting that the critical crack opening displacement is a material constant for PCO.

According to Arkhireyeva et al. [14], the values of $w_{e}, w_{e n t}$ and $w_{e y}$ are directly related to the values of $\delta_{e}, \delta_{e n t}$ and $\delta_{e y}$, respectively, according to Equations (9)-(11):

$w_{e}=\lambda \sigma_{Y} \delta_{e}$

$w_{e y}=\lambda \sigma_{Y} \delta_{e y}$

$w_{\text {ent }}=\lambda \sigma_{Y} \delta_{\text {ent }}$

where $\sigma_{Y}$ is the yield stress and $\lambda$ is the shape factor of the stress-stain curves below the yield point. The value of $\lambda$ is assumed to be 0.67 , which is attributed to the parabolic curve. The values of $w_{e}, w_{e y}$ and $w_{\text {ent }}$ estimated from COD were listed in Table 1 together with the values estimated directly from the EWF tests and the yield stress determined by the uniaxial tensile tests. The sample exhibited brittle behavior accompanied by no clear yield point at $50^{\circ} \mathrm{C}$. The values of $w_{e}$ determined from COD are coincident with the values from EWF tests at all the temperatures measured (shown in Figure 6a) though the values of $w_{e y}$ determined form COD were overestimated and the values of $w_{\text {ent }}$ were underestimated. The similar results for PEEK samples were reported by Arkhireyeva et al. [14]. 
Table 1. The yield stress $\left(\sigma_{Y}\right)$, the specific essential work $\left(w_{e}\right)$ and its components of the pre- and post-yield region $\left(w_{y}\right.$ and $w_{\text {ent }}$, respectively) estimated form EWF and COD

\begin{tabular}{|c|c|c|c|c|c|c|c|}
\hline \multirow{2}{*}{ Temp. $\left[{ }^{\circ} \mathbf{C}\right]$} & \multirow{2}{*}{$\boldsymbol{\sigma}_{\mathbf{Y}}[\mathbf{M P a}]$} & \multicolumn{2}{|c|}{$\mathbf{W}_{\mathbf{e}}\left[\mathbf{k J} \cdot \mathbf{m}^{-2}\right]$} & \multicolumn{2}{|c|}{$\mathbf{W}_{\mathbf{e y}}\left[\mathbf{k J} \cdot \mathbf{m}^{-2}\right]$} & \multicolumn{2}{c|}{$\mathbf{W}_{\mathbf{e n t}}\left[\mathbf{k J} \cdot \mathbf{m}^{-2}\right]$} \\
\cline { 3 - 8 } & & $\mathbf{E W F}$ & $\mathbf{C O D}$ & $\mathbf{E W F}$ & $\mathbf{C O D}$ & $\mathbf{E W F}$ & $\mathbf{C O D}$ \\
\hline 50 & - & 12.0 & - & 7.0 & - & 4.5 & - \\
\hline 102 & 53 & 12.0 & 13.0 & 4.0 & 6.3 & 8.1 & 6.3 \\
\hline 138 & 42 & 11.0 & 10.0 & 2.5 & 5.1 & 8.2 & 5.1 \\
\hline 150 & 38 & 9.9 & 9.1 & 1.8 & 4.5 & 8.1 & 4.5 \\
\hline 157 & 31 & 8.1 & 7.5 & 1.8 & 3.7 & 6.3 & 3.7 \\
\hline
\end{tabular}

Furthermore, we examined the temperature dependence of the slope $\alpha(T)$ of the $\delta_{n t} L$ relation. As shown in Figure 8, the inverse of $\ln \alpha(T)$ was found to be proportional to the inverse of $T_{g}-T$. Thus, the temperature dependence of the slope $\alpha(T)$ becomes according to Equation (12):

$$
\ln \alpha(T)=\frac{-c_{1}\left(T-T_{g}\right)}{c_{2}+T-T_{g}}
$$

where $c_{1}=3.82$ and $c_{2}=-61.8 \mathrm{~K}$. It is very interesting to note that the empirical equation is the WLF type. Consequently, the empirical relation Equation (12) makes it possible to estimate the displacements at yield and break at any temperature.

The polarized IR spectra were measured simultaneously with the tensile stress as a function of tensile time or displacement. Smaller DENT specimens with $15 \mathrm{~mm}$ width were used for the measurements and the distance between two clamps was $15 \mathrm{~mm}$. Figure 9 compares the work of fracture between EWF and rheo-EWF tests. As seen in this figure, it

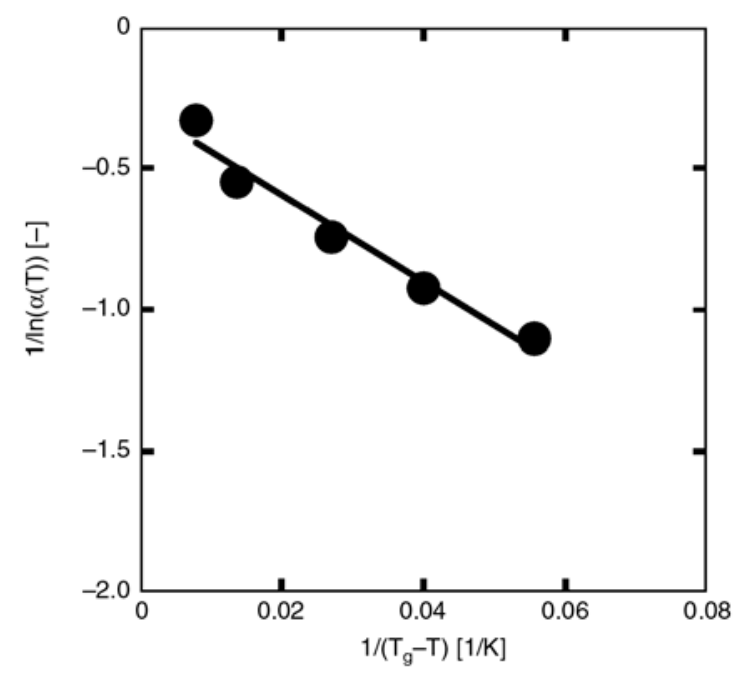

Figure 8. WLF plot of $\alpha(T)$ against $T_{g}-T . \alpha(T)$ represents the slope of displacement of necking-tearing region $\left(\delta_{n t}\right)$ against the ligament length was confirmed that the fracture data obtained from rheo-EWF tests are almost consistent with those of the EWF tests. The data of orientation function measured simultaneously with EWF tests are shown in Figure 10 where both the load and the value of orientation function $f$ are plotted against displacement at 110 and $170^{\circ} \mathrm{C}$. The orientation function $f$ was around zero up to the tearing start point and linearly increased with increasing the elongation. In the yielding in the ligament region almost no molecular chains orient to the stretching direction, indicating that large scale molecular mobility of molecular chains is not activated until ligament full-yielding. This suggests that the agglomeration of chain molecules act as a deformation unit and the work for yielding is consumed by the release of the agglomeration of chain molecules. On the other hands, the necking or tearing process induce the molecular orientation and the increase in molecular orientation function will be due to the expansion of necked region in the plastic zone. The plastic work for expanding the necking

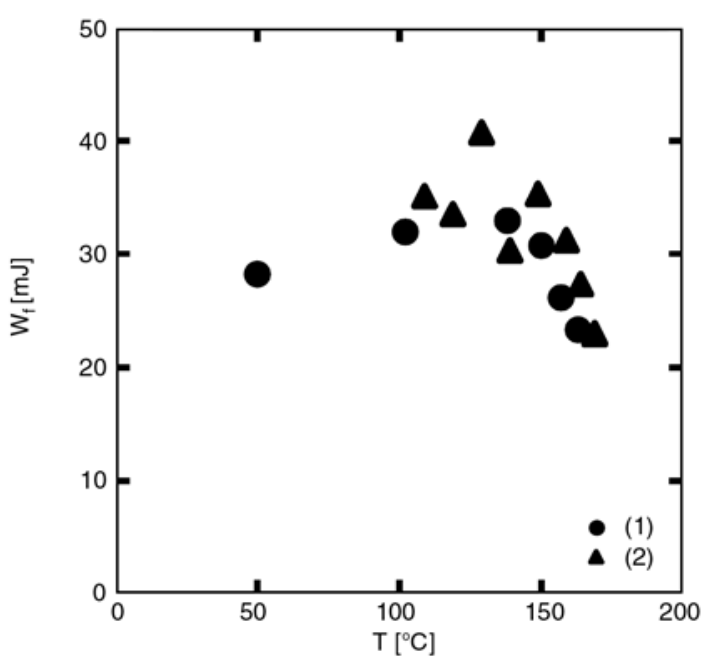

Figure 9. The temperature dependence of total work $\left(W_{f}\right)$ for 2 samples with different size. (1): the sample for the EWF tests and (2): the sample for the rheo-optical EWF tests 

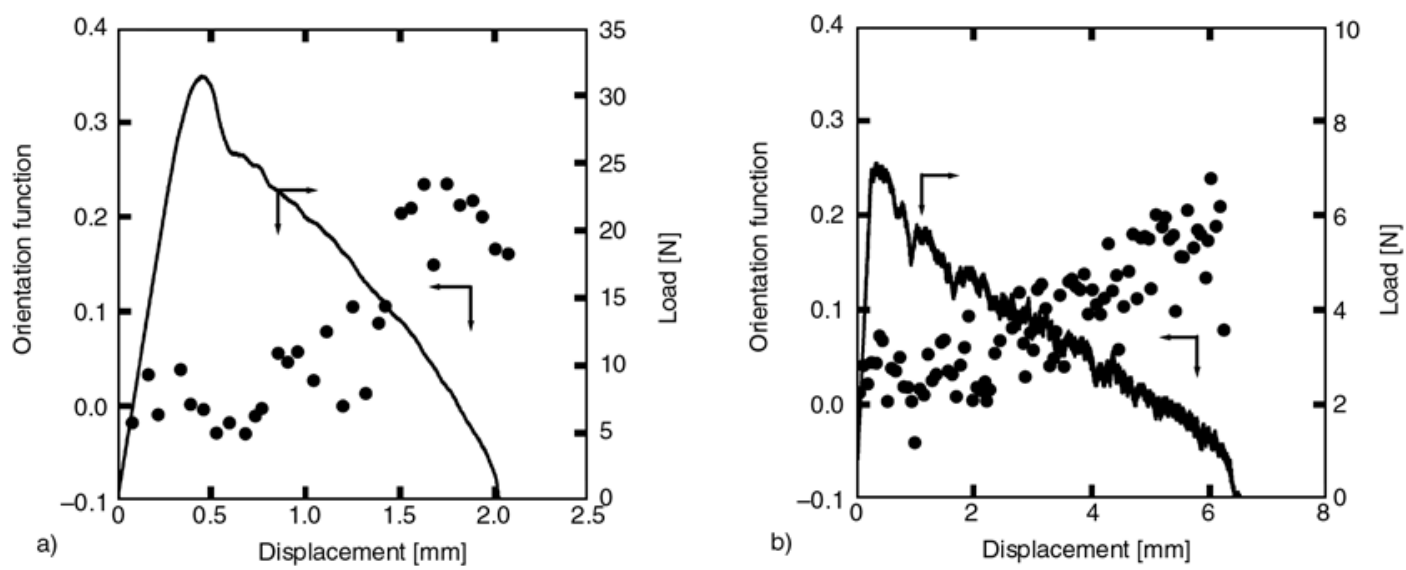

Figure 10. The load vs. displacement curves with simultaneous orientation function measurements of the DENT specimens at $110^{\circ} \mathrm{C}$ (a) and $170^{\circ} \mathrm{C}(\mathrm{b})$

and/or tearing positively corresponds to the elongation sensitivity of molecular orientation, indicating that the work for the plastic deformation is consumed almost by the expansion of the molecular orientated (micro-necked) portions to the plastic plane.

\section{Conclusions}

The fracture toughness of polyolefin of a glassy polycyclo-olefin (PCO) was investigated between room temperature and near $T_{g}$ by the essential work of fracture method using double-edge notched tensile specimens.

It was shown that the PCO follows the EWF concept in the temperature range between 50 and $157^{\circ} \mathrm{C}$ where the ligament yielding appear at a maximum point on the stress-displacement curves and subsequently the necking and tearing processes take place in the post yielding region. The essential work of fracture, $w_{e}$ required for the ligament yielding drops as the temperature approaches $T_{g}$. The non-essential work of fracture, $w_{p}$ attributed to tearing process after yielding is consumed to expand the plastic region and causes molecular chains to orient to the stretching direction.

A linear relationship was confirmed between the total specific work of fracture and ligament length over the entirely experimental temperature range. It was found that although specific essential work of fracture decreases with increasing temperature and the specific non-essential work of fracture increased with increasing temperature but dropped at near $T_{g}$. A linear relationship was also found for yielding and necking/tearing components of the total specific work of fracture as a function of ligament length. These specific essential work components were found to be dependent of temperature whilst the yielding component decreased with temperature. The contribution of necking/tearing component was substantially greater than that of the yielding component. Similarly, the displacement at fracture $\delta_{b}$ can be divided into the yield displacement and tearing component and the displacements were proportional to the ligament length. In addition, it was found that the temperature dependence of the displacement at break follows the WLF type of equation.

According to polarized FT-IR data measured simultaneously with EWF tests, the molecular orientation started in the post-yielding, indicating that the work for the plastic deformation is consumed almost by the expansion of the molecular orientated (micro-necked) portions to the plastic plane.

\section{References}

[1] Kuno T., Kawamura T., Nitta K.: Mechanical properties of polycyclo-olefins in the vicinity of the glass transition temperature. Polymer Bulletin, 59, 847-854 (2008).

[2] Broberg K. B.: On stable crack growth. Journal of the Mechanics and Physics of Solids, 23, 215-237 (1975).

[3] Cotterell B., Reddel J. K.: The essential work of plane stress ductile fracture. International Journal of Fracture, 13, 267-277 (1977).

[4] Ward I. E., Sweeney J.: An introduction to the mechanical properties of solid polymers. Wiley and Sons, West Sussex (2004).

[5] Haward R. N., Young R. J.: The physics of glassy polymers. Chapman \& Hall, London (1997). 
[6] Wu J., Mai Y-W.: The essential fracture work concept for toughness measurement of ductile polymers. Polymer Engineering and Science, 36, 2275-2288 (1996).

[7] Karger-Kocsis J., Czigány T., Moskala E. J.: Deformation rate dependence of the essential and nonessential work of fracture parameters in an amorphous copolyester. Polymer, 39, 3939-3944 (1998).

[8] Arkhireyeva A., Hashemi S.: Effect of temperature on fracture properties of an amorphous poly(ethylene terephthalate) (PET) film. Journal of Materials Science, 37, 3675-3683 (2002).

[9] Ferrer-Balas D., Maspoch M. L., Martinez A. B., Santana O. O.: On the essential work of fracture method: Energy partitioning of the fracture process in iPP films. Polymer Bulletin, 42, 101-108 (1999).

[10] Mai Y-W., Cotterell B., Horlyck R., Vigna G.: The essential work of plane stress ductile fracture of linear polyethylenes. Polymer Engineering and Science, 27, 804-809 (1987).

[11] Mai Y-W., Powell P.: Essential work of fracture and Jintegral measurements for ductile polymers. Journal of Polymer Science, Part B: Polymer Physics, 29, 785-793 (1991).

[12] Hashemi S.: Temperature dependence of work of fracture parameters in polybutylene terephthalate (PBT). Polymer Engineering and Science, 40, 1435-1446 (2000).

[13] Bárány T., Karger-Kocsis J., Czigány T.: Effect of hygrothermal aging on the essential work of fracture response of amorphous poly(ethylene terephthalate) sheets. Polymer Degradation and Stability, 82, 271278 (2003)

[14] Arkhireyeva A., Hashemi S.: Effect of temperature on work of fracture parameters in poly(ether-ether ketone) (PEEK) film. Engineering Fracture Mechanics, 71, 789-804 (2004).
[15] Karger-Kocsis J., Bárány T., Moskala E. J.: Plane stress fracture toughness of physically aged plasticized PETG as assessed by the essential work of fracture (EWF) method. Polymer, 44, 5691-5699 (2003).

[16] Bárány T., Ronkay F., Karger-Kocsis J., Czigány T.: In-plane and out-of-plane fracture toughness of physically aged polyesters as assessed by the essential work of fracture (EWF) method. International Journal of Fracture, 135, 251-265 (2005).

[17] Bárány T., Földes E., Czigány T.: Effect of thermal and hygrothermal aging on the plane stress fracture toughness of poly(ethylene terephthalate) sheets. Express Polymer Letters, 1, 180-187 (2007).

[18] Karger-Kocsis J., Moskala E. J.: Toughness response of amorphous (co)polyesters using the essential work of fracture approach. ANTEC Papers, 60, 1751-1755 (2002).

[19] Ke B.: New methods of polymer characterization. Wiley, New York (1968).

[20] Nitta K., Okamoto K., Yamaguchi M.: Mechanical properties of binary blends of polypropylene with ethylene- $\alpha$-olefin copolymer. Polymer, 39, 53-58 (1998).

[21] Zbindem R.: Infrared spectroscopy of high polymers. Academic Press, New York (1964).

[22] Onogi S., Asada T., Tanaka A.: Rheo-optical studies of high polymers. XIV. Study of the deformation mechanism in polymer blends of polypropylene with ethylene-propylene rubber. Journal of Polymer Science Part A-2: Polymer Physics, 7, 171-182 (1969).

[23] Mouzakis D. E., Karger-Kocsis J., Moskala E. J.: Interrelation between energy partitioned work of fracture parameters and the crack tip opening displacement in amorphous polyester films. Journal of Materials Science Letters, 19, 1615-1619 (2000). 